\title{
Bowel preparation in pediatric colonoscopy: results of an open observational study
}

Authors

Institutions
Thomas Berger ${ }^{1}$, Martin Classen ${ }^{2}$, Harald Engelhardt ${ }^{3}$, Klaus-Michael Keller ${ }^{4}$, Martin W. Laass ${ }^{5}$, Ralph Melchior ${ }^{6}$, Carsten Posovszky ${ }^{7}$, Burkhard Rodeck ${ }^{8}$, Katharina Schaper ${ }^{9}$, Rolf Behrens ${ }^{10}$

Institutions are listed at the end of article. submitted: 8 . April 2015 accepted after revision: 25. April 2016

\section{Bibliography}

DOI http://dx.doi.org/

10.1055/s-0042-107789

Published online: 2.6.2016

Endoscopy International Open 2016; 04: E820-E827

(c) Georg Thieme Verlag KG Stuttgart · New York

E-ISSN 2196-9736

\section{Corresponding author}

Thomas Berger, MD

Vestische Kinder- und Jugendklinik Datteln Universität Witten-Herdecke Dr. Friedrich-Steiner-Str. 5 D-45711 Datteln Germany

Fax: +49(0)2363-975255 t.berger@kinderklinik-datteln. de

\section{License terms}

Background and study aims: The goal of this study was to analyze the bowel cleansing methods currently used for pediatric colonoscopy in terms of effectiveness, tolerance and safety.

Patients and methods: Data from 768 colonoscopies reported by 28 centers were registered in an online database for further analysis. Binary logistic regression was used to determine how preparation methods affected the cleaning effect (Aronchick score) and the rate of adverse events (Aes) and complications.

Results: The most frequently reported cleansing agents were sodium picosulphate $(54.2 \%)$ and polyethylene-glycol (41.3\%) in various combinations. The cleaning effect was good to excellent in $72.6 \%$ of patients. AEs during the preparation period occurred in $21.5 \%$ of patients. Complica-

\section{Introduction}

$\nabla$

Preparation and bowel cleansing represent an important step in conducting colonoscopy. A well prepared and clean bowel is absolutely necessary for making an accurate diagnosis. Insufficient cleaning makes the procedure more time consuming, technically more difficult or even impossible with a higher risk of complications [1, 2].

An ideal preparation for colonoscopy should be simple, fast, effective, safe, and well tolerated. There are some particular issues concerning safety and tolerance in childhood: Many of the commonly applied regimens use agents that are not approved for children. To date only one agent, Picoprep $^{\circledR}$, has recently received official approval in Germany for bowel preparation in children. The demand from families and health care institutions for a short preparation time leads to the necessity to ingest a high volume of liquids in a short period of time. Thus tolerability is limited and problems such as vomiting or refusal of further oral intake are common [3-8]. Gastric tubes may be necessary for application [6]. tions during endoscopy were reported in $12.1 \%$ and were mostly mild. The different agents had no influence on the cleaning effect. In contrast the risk of AEs during preparation was significantly increased when polyethylene-glycol was used (odds ratio (OR) 2.112, $P=0.002$ ) but reduced with the use of sodium picosulphate (OR $0.380, P<0.001)$. In particular, the risk of needing a nasogastric tube to complete clean-out was about 10 -fold higher when polyethylene-glycol was used.

Conclusions: A large variety of regimens are used for bowel preparation in children. We found a good overall cleaning result independent of the agents used. Cleansing agents, on the other hand, had a significant influence on tolerance and safety.

There are relatively few good data about the effectiveness and tolerance of preparation regimens available for children. In 2010 Hunter and Mamula reviewed the literature, comprising 8 randomized clinical trials, and stated: "Based on the available data it is difficult to make precise comparisons..." and "...the existing agents require further evaluation regarding their safety and dose profiles that provides appropriate preparations with minimal adverse events." [9]. The results of the studies published after conclusion of this review do not change this overall image [3,7,8, 10-12]. The authors of the only existing evidence-based recommendations conclude: "There is no ideal bowel cleansing regimen and, thus, numerous cleanout protocols are in use." [13]. As no other evidence-based guidelines exist, the search for an ideal preparation regimen still continues.

There is no detailed information about the regimens used for bowel cleansing especially in Germany. The methods used are heterogeneous and seem to depend more on personal preferences and local habits and conditions with a low degree of standardization. We therefore have chosen the 
concept of an open observational study to pursue two objectives: First, to obtain a realistic picture of the different methods and agents used in Germany and second, to try to analyze whether these "real-life" regimens differ in terms of effectiveness and safety.

\section{Patients and methods \\ $\nabla$}

\section{Data collection}

In this study we analyzed data from a multicenter online register that was created in the context of a quality management project of the Society of Pediatric Gastroenterology and Nutrition (GPGE). Participation was voluntary and participants were asked to enter information about all colonoscopies carried out in children from 0 to 18 years from October 2011 to November 2012. A dataset was created for each individual colonoscopy. Each dataset contained items concerning structure, process and outcome ( Table 1). We did not specify any standard or protocol concerning the agents to be used. The choice of the cleansing regimen was completely left to the discretion of the participating physicians. No identifying patient information was transmitted to the database. A total of 768 datasets were provided by 28 participating centers.

The Aronchick score was applied for assessment of the cleaning effect. This score is validated and simple to use ( Table 2) [14]. The alternative Ottawa/Boston score is based on assessment of 3 segments of the colon applying a scale of 0 to 3 points to each of them, whereas application of the Aronchick score only requires a global assessment of the colon as a whole, applying a scale of 5 levels. Both scores are validated and suitable for the assessment of bowel preparation. The 2015 American Society for Gastrointestinal Endoscopy guideline states: "The Aronchik Scale is a global rating best suited for comparing different bowel preparations because it assesses the quality of the preparation encountered during the initial inspection of the colon."[15] In the context of our survey it was simpler to introduce the Aronchick score for 2 reasons. First, we were neither able to train the participating centers in applying the score nor to control its use independently. These factors then favored the use of the simpler Aronchick score. Second, for our statistical analyses,we had to dichotomize the cleaning effect into 2 categories (good cleaning effect/bad cleaning effect), so that the more detailed Ottawa/Boston score would not have provided an advantage in the context of our study.

Adverse events (AEs) during the preparation phase and complications during the examination itself were recorded based on predetermined categories. AEs during the preparation period were categorized as follows: no AE; nasogastric tube placement; vomiting; abdominal pain; and refusal of further oral intake. Examination time was recorded in minutes, separately until the cecum and the terminal ileum were reached. Categories for complications during the endoscopy procedure were: no complications; minor complications; and major complications, and included events such as technical problems, bleeding, perforation, infection, arterial hypotension, oxygen desaturation, and death.

\section{Statistical analyses}

In the first step we aimed to obtain a general view of the methods used for bowel preparation and analyzed the data with descriptive methods reporting percentages, mean values, and medians. To evaluate the applied regimens in regard to their effectiveness and safety we tried to establish a correlation between the data

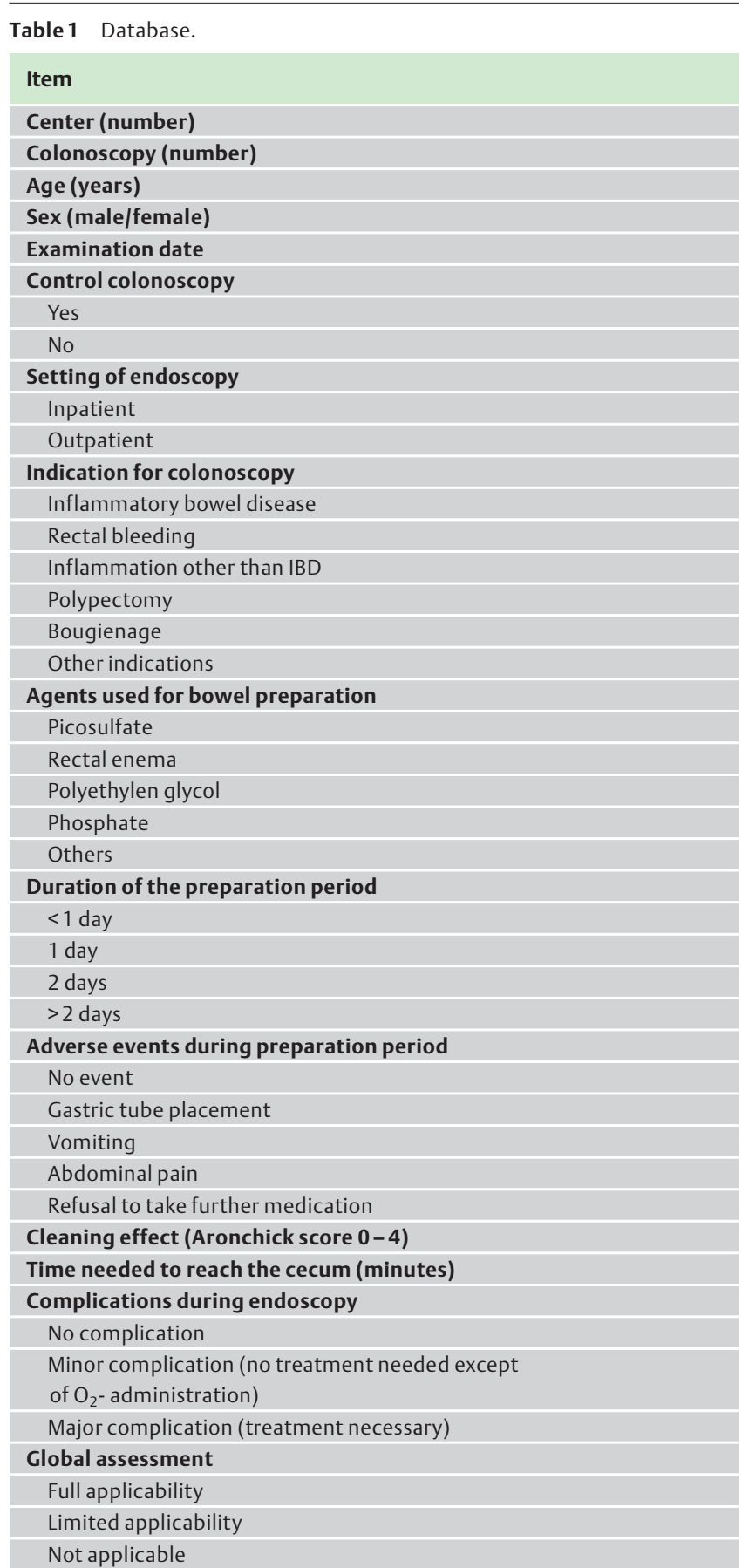

Table 2 Aronchick score.

\begin{tabular}{|l|l|}
\hline Aronchick score: & Cleaning effect \\
\hline 0 Excellent & $\begin{array}{l}\text { Small volume of clear liquid or greater than } 95 \% \\
\text { of surface seen }\end{array}$ \\
\hline 1 Good & $\begin{array}{l}\text { Large volume of clear liquid covering } 5 \% \text { to } 25 \% \\
\text { of surface but greater than } 90 \% \text { of surface seen }\end{array}$ \\
\hline 2 Fair & $\begin{array}{l}\text { Some semi-solid stool that could be suctioned or } \\
\text { washed away but greater than } 90 \% \text { of surface seen }\end{array}$ \\
\hline 3 Poor & $\begin{array}{l}\text { Semi-solid stool that could not be } \\
\text { suctioned or washed away and less than } 90 \% \text { of } \\
\text { surface seen }\end{array}$ \\
\hline 4 Inadequate & \begin{tabular}{l} 
Termination and need for repreparation \\
\hline
\end{tabular}
\end{tabular}


Original article

\begin{tabular}{|c|c|c|}
\hline Characteristic & $\mathbf{n}$ & $\%$ \\
\hline Age. years. median (range) & $13(0-18)$ & \\
\hline Sex (male : female) & $412: 356$ & $54: 46$ \\
\hline \multicolumn{3}{|l|}{ Setting of endoscopy } \\
\hline Inpatient & 666 & 86.7 \\
\hline Outpatient & 102 & 13.3 \\
\hline \multicolumn{3}{|l|}{ Indication for colonoscopy } \\
\hline Inflammatory bowel disease & 472 & 61.5 \\
\hline Rectal bleeding & 109 & 14.2 \\
\hline Inflammation other than IBD & 98 & 12.8 \\
\hline Polypectomy & 32 & 4.2 \\
\hline Bougienage & 2 & 0.3 \\
\hline Other indications & 55 & 7.2 \\
\hline Agents used for bowel preparation & $\begin{array}{l}\text { Total/ } \\
\text { with rectal enema }\end{array}$ & \\
\hline Picosulphate & $416 / 183$ & $54.2 / 23.8$ \\
\hline Polyethylene glycol (PEG) & $317 / 87$ & $41.3 / 11.3$ \\
\hline Sodium phosphate & $71 / 52$ & $9.2 / 6.8$ \\
\hline Others & $96 / 51$ & $12.5 / 6.6$ \\
\hline Rectal enema only & 31 & 4.0 \\
\hline \multicolumn{3}{|l|}{ Duration of the preparation period } \\
\hline$<1$ day & 194 & 25.3 \\
\hline 1 day & 484 & 63.0 \\
\hline 2 days & 85 & 11.1 \\
\hline$>2$ days & 5 & 0.7 \\
\hline \multicolumn{3}{|l|}{ Adverse events during preparation period } \\
\hline No event & 603 & 78.5 \\
\hline Any event ${ }^{*}$ & 165 & 21.5 \\
\hline Gastric tube placement & 107 & 13.9 \\
\hline Vomiting & 64 & 8.3 \\
\hline Abdominal pain & 24 & 3.1 \\
\hline Refusal to take further medication & 9 & 1.2 \\
\hline \multicolumn{3}{|l|}{ Cleaning effect (Aronchick score) } \\
\hline 0 & 312 & 40.6 \\
\hline 1 & 246 & 32.0 \\
\hline 2 & 147 & 19.1 \\
\hline 3 & 58 & 7.6 \\
\hline 4 & 5 & 0.7 \\
\hline \multicolumn{3}{|l|}{ Time needed to reach the cecum } \\
\hline Minutes. mean (+/-SD) & $15.5(+/-9.2)$ & \\
\hline Cecum not reached & 38 & 4.9 \\
\hline \multicolumn{3}{|l|}{ Complications during endoscopy } \\
\hline No complication & 675 & 87.9 \\
\hline Minor complication (no treatment needed except of $\mathrm{O}_{2}$ administration)* & 87 & 11.3 \\
\hline Major complication (treatment necessary)* & 6 & 0.8 \\
\hline \multicolumn{3}{|l|}{ Global assessment } \\
\hline Full applicability & 719 & 93.6 \\
\hline Limited applicability & 45 & 5.9 \\
\hline Not applicable & 4 & 0.5 \\
\hline
\end{tabular}

Table 3 Patient characteristics $(n=768)$.

* There may be more than one complication per procedure

concerning methods and results using regression models. Factors influencing the cleaning effect, AEs, and complications were calculated on the basis of binary logistic regression. For that purpose 2 additional structural parameters were calculated to characterize the participating centers: 1) the total number of reported endoscopies; and 2) the percentage of outpatients. The outcome parameter "cleaning effect" measured by the Aronchick score was dichotomized into 2 categories: good cleaning effect (Aronchick score 0 to 1 ) and bad cleaning effect (score 2 to 4 ). Events and complications were divided into two categories: no event/ complication; any event/complication. Further subanalyses were performed for each single type of event during the preparation period. Factors influencing the examination time were investigated in a similar way by linear regression using the time needed to reach the cecum as a reference.

Software packages MS-Excel (2010) and SPSS (IBM ${ }^{\circledR}$ SPSS $^{\circledR}$ statistics, v.19\&21) were used for all statistical analyses. Access to the database was password-protected. Each participating center had access only to its individual data. The design of this project was strictly observational with no experimental investigation. Routine written informed consent regarding the colonoscopy procedure was obtained from parent(s). The study was approved by the ethics committee of the University of Witten-Herdecke. No external funding was required. 


\section{Results}

$\nabla$

\section{Descriptive analyses}

We were able to analyze data from a total of 768 colonoscopies reported by 28 participating centers ( $\bullet$ Table 3 ). The median age of the patients was 13 years (IQR 10-15). The most frequent indication reported for colonoscopy was inflammatory bowel disease; the other indications are detailed in 0 Table3. 0 Table 3 also summarizes the cleansing agents used, the most frequently reported being sodium picosulphate and polyethylene-glycol (PEG). These were applied in a number of different combinations ( $\bullet$ Table 4). Usually patients are given a clear liquid diet at least for some time during the preparation period. In our database this item was originally included and a clear liquid diet was documented in 295 patients (38.4\%) in our survey. When we discussed the results with our participating centers, this rate turned out to be far too low, mainly due to the difficulty to define such a diet consistently. So we decided to exclude this aspect from further analysis. Results of inpatients compared with outpatients are shown in $\bullet$ Table 5 .

The median duration of the bowel preparation period was 1 day. Of the colonoscopies, $13.3 \%$ were prepared and performed in an outpatient setting. The cleaning effect defined by the Aronchick score varied significantly among the different centers. Bowel cleaning was good or excellent in $72.7 \%$ of patients and only 0.7 $\%$ of the colonoscopies had to be terminated due to insufficient cleaning. AEs during the preparation phase occurred in $21.5 \%$ of cases. These consisted mainly of the necessity to use a nasogastric tube ( $n=107,13.9 \%$; for more detail see $\bullet$ Table 3 ). Completion rate was high with the cecum reached in $95.1 \%$ of patients. Complications during the endoscopy procedure were reported in $12.1 \%$ and were mostly mild. Serious complications were reported in 6 cases $(0.8 \%)$ : a drop in blood pressure $(n=2)$, perforation $(n=2)$, hemorrhage $(n=1)$, oxygen desaturation $(n=1)$. Perforation occurred in a patient with severe intestinal graft versus host disease and in a second patient after balloon dilation of a high-grade stricture.

\section{Regression models}

The results of the regression analyses are shown in $\bullet$ Table6. The regression model as a whole did not show that the measures taken nor the duration of the preparation phase had any marked influence on the cleaning effect. In particular, no influence of the choice of cleansing agents could be found. The low value of $\mathrm{Na}-$ gelkerke's $R^{2}(0.059)$ indicates that only a small part of the variance can be explained by the model.

In contrast, we found a clear link between the preparation methods and the frequency of AEs during the preparation period. Nagelkerke's $R^{2}$ reached an acceptable value of 0.277 for this part of the model. The risk of events is increased when PEG is used (OR 2.112) and reduced when sodium picosulphate is used ( $O R$ 0.380 ). Further analysis showed that the risk of insertion of a nasogastric tube was 10-fold higher when PEG was used whereas the risk of nasogastric tubes, vomiting, and refusal were significantly reduced with the use of sodium picosulphate $(\bullet$ Table6c) Regression models looking at complications during the examination showed a significant influence only with regard to parameters concerning the setting of the endoscopy (procedure on an outpatient base: OR 0.4). Overall subjective assessment was influenced only by the cleaning effect. Linear regression $(\bullet$ Table 7) showed that the duration of endoscopy in our sample was significantly influenced by the cleaning effect (Aronchick score,
Table 4 Combinations of bowel cleansing agents.

\begin{tabular}{|lcc|}
\hline & $\begin{array}{l}\text { Without } \\
\text { rectal } \\
\text { enema }\end{array}$ & $\begin{array}{c}\text { With } \\
\text { rectal } \\
\text { enema }\end{array}$ \\
\hline PEG only & 143 & 58 \\
\hline PEG + picosulphate & 59 & 4 \\
\hline PEG + sodium phosphate & 0 & 3 \\
\hline PEG + other agents* & 25 & 16 \\
\hline PEG + picosulphate + sodium phosphate & 0 & 0 \\
\hline PEG + picosulphate + other agents & 1 & 0 \\
\hline PEG + sodium phosphate + other agents & 2 & 5 \\
\hline PEG + picosulphate + sodium phosphate & 0 & 1 \\
\hline +other agents & 173 & 167 \\
\hline Picosulphate only & 0 & 0 \\
\hline Picosulphate + sodium phosphate & 0 & 1 \\
\hline Picosulphate + other agents & 0 & 10 \\
\hline Picosulphate + sodium phosphate + other & & \\
\hline agents & 4 & 26 \\
\hline Sodium phosphate only & 13 & 7 \\
\hline Sodium phosphate + other agents & 1 & 11 \\
\hline Other agents only & - & 31 \\
\hline Rectal enema only & 7 & - \\
\hline No preparation & 768 & \\
\hline Total: & & \\
\hline "Oth a & & \\
\hline
\end{tabular}

* "other agents" mainly consisted of bisacodyl and senna

Table 5 Comparison of inpatients and outpatients.

\begin{tabular}{|lrrrr|}
\hline & inpatients & outpatients \\
\hline $\mathbf{n}=$ & $\mathbf{6 6 6}$ & & \multicolumn{1}{l|}{$\mathbf{1 0 2}$} \\
\hline Indication for colonoscopy & & & & \\
\hline Inflammatory bowel disease & 408 & $61.3 \%$ & 58 & $56.9 \%$ \\
\hline Rectal bleeding & 89 & $13.4 \%$ & 15 & $14.7 \%$ \\
\hline Inflammation other than IBD & 83 & $12.5 \%$ & 14 & $13.7 \%$ \\
\hline Polypectomy & 24 & $3.6 \%$ & 8 & $7.8 \%$ \\
\hline Bougienage & 2 & $0.3 \%$ & 0 & $0.0 \%$ \\
\hline Other indications & 60 & $9.0 \%$ & 7 & $6.9 \%$ \\
\hline Preparation & & & & \\
\hline PEG & 310 & $46.5 \%$ & 8 & $7.8 \%$ \\
\hline Pico & 329 & $49.4 \%$ & 87 & $85.3 \%$ \\
\hline Phosphate & 71 & $10.7 \%$ & 0 & $0.0 \%$ \\
\hline Others & 86 & $12.9 \%$ & 7 & $6.9 \%$ \\
\hline Rectal enema & 314 & $47.1 \%$ & 26 & $25.5 \%$ \\
\hline Results & & & & \\
\hline Adverse events during preparation & 159 & $23.9 \%$ & 7 & $6.9 \%$ \\
\hline Good cleaning effect & 478 & $71.8 \%$ & 80 & $78.4 \%$ \\
\hline Complications during endoscopy & 82 & $12.3 \%$ & 12 & $11.8 \%$ \\
\hline
\end{tabular}

coefficient B (coeff.) $0.756(0.066-1.446), P=0.03)$, the use of PEG (coeff. $1.631(0.255-3.007), P=0.02)$, and the use of sodium phosphate (coeff. $-3.207, P<0.01$ ). However, it should be noted that the last 3 models mentioned resulted in only low $\mathrm{r}^{2}$ values and thus did not yield convincing results as a whole.

\section{Discussion}

Our analysis shows a very heterogeneous picture of the regimens used for the preparation of colonoscopy in children. The cleansing agents, duration of the preparation period, and setting (inpatient or outpatient) vary significantly. The most frequently used 
Table 6 Binary logistic regression analysis of the association between variables and outcome parameters of bowel preparation.

\begin{tabular}{|c|c|c|}
\hline & OR $(95 \% \mathrm{Cl})$ & $P$ \\
\hline \multicolumn{3}{|c|}{$\begin{array}{l}\text { a) Cleaning effect: factors associated with a good cleaning effect (Aronchick } 0-1 \text { ); } \\
\text { Nagelkerke's } R^{2}: 0.059\end{array}$} \\
\hline Percentage of outpatients in center & $1.015(1.005-1.025)$ & 0.003 \\
\hline Age (years) & $0.938(0.902-0.975)$ & 0.001 \\
\hline \multicolumn{3}{|c|}{$\begin{array}{l}\text { b) Adverse events during preparation period: factors associated with the occurrence of any event; } \\
\text { Nagelkerke's } R^{2}: 0.277\end{array}$} \\
\hline Percentage of outpatients in center & $0.982(0.966-0.997)$ & 0.022 \\
\hline Polyethylene glycol & $2.112(1.303-3.422)$ & 0.002 \\
\hline Picosulphate & $0.380(0.239-0.604)$ & $<0.001$ \\
\hline \multicolumn{3}{|c|}{$\begin{array}{l}\text { c) Adverse events during preparation period: factors associated with the occurrence of gastric tube placement; } \\
\text { Nagelkerke's } R^{2}: 0.574\end{array}$} \\
\hline Number of colonoscopies performed in center & $1.042(1.030-1.054)$ & $<0.001$ \\
\hline Percentage of outpatients in center & $0.934(0.892-0.979)$ & 0.004 \\
\hline Age (years) & $0.897(0.843-0.953)$ & $<0.001$ \\
\hline Polyethylene glycol & $10.196(3.686-28.202)$ & $<0.001$ \\
\hline Picosulphate & $0.240(0.109-0.526)$ & $<0.001$ \\
\hline \multicolumn{3}{|c|}{$\begin{array}{l}\text { d) Adverse events during preparation period: factors associated with the occurrence of vomiting; } \\
\text { Nagelkerke's } R^{2}: 0.05\end{array}$} \\
\hline Picosulphate & $0.435(0.248-0.763)$ & 0.004 \\
\hline Sodium Phosphate & $2.084(1.044-4.162)$ & 0.037 \\
\hline \multicolumn{3}{|c|}{$\begin{array}{l}\text { e) Adverse events during preparation period: factors associated with the occurrence of abdominal pain; } \\
\text { Nagelkerke's } R^{2}: 0.072\end{array}$} \\
\hline Number of colonoscopies performed in center & $0.979(0.963-0.996)$ & 0.016 \\
\hline Other agents & $2.779(1.130-6.832)$ & 0.026 \\
\hline \multicolumn{3}{|c|}{$\begin{array}{l}\text { f) Adverse events during preparation period: factors associated with refusal of further oral intake; } \\
\text { Nagelkerke's } R^{2}: 0.127\end{array}$} \\
\hline Picosulphate & $0.104(0.013-0.834)$ & 0.033 \\
\hline Rectal enema & $4.484(0.921-21.833)$ & 0.063 \\
\hline \multicolumn{3}{|c|}{$\begin{array}{l}\text { g) Complications during colonoscopy: factors associated with the occurrence of any complication; } \\
\text { Nagelkerke's } R^{2}: 0.024\end{array}$} \\
\hline Percentage of outpatients in center & $1.020(1.008-1.032)$ & 0.001 \\
\hline Performance of colonoscopy in an outpatient setting & $0.400(0.170-0.942)$ & 0.036 \\
\hline \multicolumn{3}{|c|}{$\begin{array}{l}\text { h) Overall subjective assessment by examiner: factors associated with full applicability; } \\
\text { Nagelkerke's } R^{2}: 0.374\end{array}$} \\
\hline Aronchick score 1 & $1.186(0.331-4.251)$ & 0.793 \\
\hline Aronchick score 2 & $0.341(0.116-1.001)$ & 0.050 \\
\hline Aronchick score 3 & $0.023(0.009-0.059)$ & $<0.001$ \\
\hline Aronchick score 4 & $0.005(0.000-0.051)$ & $<0.001$ \\
\hline
\end{tabular}

agents in this study were sodium picosulphate and PEG in various combinations.

Participation in our project was voluntary. The participating centers in our study represent a relatively balanced sample of institutions active in pediatric gastroenterology in Germany. Institutions differing in size and level of specialization as well as performing endoscopy both on an inpatient and outpatient setting were represented. Both the relatively low number of procedures per center and the relatively high rate of inpatients are in part due to some structural peculiarities: Most activities take place in hospitals rather than in physicians' offices. Furthermore, pediatric gastroenterology in Germany is less centralized than in other European countries where procedures are only performed in a limited number of larger institutions.

Centers were asked to transmit data from all colonoscopies during the project period. In a different survey prior to this study, members of the GPGE were asked to indicate their total number of colonoscopies per year without specific details (unpublished data). The number of procedures in centers participating in both projects was similar. While we cannot completely be sure that no procedures were omitted, we assume that the number of unreported endoscopies was not significant. All centers, whether large or small, performed accepted standardized procedures. Our data showed that the number of colonoscopies performed by a center did not influence the quality of bowel preparation. In comparison with inpatients, outpatients had similar indications for colonoscopy. They were more often prepared with picosulfate than with PEG or sodium phosphate and received fewer rectal enemas. No difference in cleaning effect could be found but outpatients seemed to have had fewer AEs during the preparation period. However, that may be due, in part, to selection and reporting bias.

Table 7 Linear regression analysis of association between variables and duration of colonoscopy (time to cecum).

\begin{tabular}{|c|c|c|c|c|}
\hline \multirow[t]{2}{*}{$R^{2}: 0.029$} & \multirow[t]{2}{*}{ coefficient B } & \multicolumn{2}{|l|}{$95 \% \mathrm{Cl}$} & \multirow[t]{2}{*}{$P$} \\
\hline & & $\begin{array}{l}\text { Lower } \\
\text { limit }\end{array}$ & $\begin{array}{l}\text { Upper } \\
\text { limit }\end{array}$ & \\
\hline Sodium phosphate & -3.207 & -5.501 & -0.913 & 0.006 \\
\hline Polyethylene glycol & 1.631 & 0.255 & 3.007 & 0.020 \\
\hline $\begin{array}{l}\text { Cleaning effect } \\
\text { (Aronchick score) }\end{array}$ & 0.756 & 0.066 & 1.446 & 0.032 \\
\hline
\end{tabular}


The search for factors that influence the outcome of the preparation period was the main objective of this analysis. The outcome in this context consists of 2 elements: the effectiveness as measured by the cleaning effect and the tolerability and safety as measured by the rate of AEs and complications.

The cleaning effect, on the one hand, varied markedly among the centers. This variability, on the other hand, could not be explained reasonably on the basis of the recorded factors. The concomitant regression model only achieves a low quality of prediction and shows no significant influence of the agents used. This implies that influencing factors outside the model must play an important role here.

In contrast, we found that the regimens clearly influenced tolerability and acceptance of the preparation. That was particularly true for the rate at which nasogastric tubes were needed or used. In this study, the placement of a nasogastric tube was labelled as an AE. Even though it might be a fixed part of the preparation procedure in some centers, we consider the rate of gastric tube placements an indicator of tolerability of the agents used. This rate was many times higher when PEG was used as an element of the preparation. Additional correlations were found for the outcomes "vomiting" and "refusal to take the oral agent". For all 3 aspects of tolerability and acceptance, use of sodium picosulphate had a significant positive effect. In contrast, from our data, no influence on tolerability could be attributed to patient age, indication for colonoscopy, or duration of the preparation period.

The fact that only $72 \%$ of patients had a very good cleaning result could be considered as insufficient. However, comparability of the cleaning effect in published studies is limited as no consistent scoring system is used by differing authors. Some studies specify the cleaning result with scoring systems similar to the Aronchick score, reporting good to excellent cleaning results with rates ranging from $40 \%$ to $100 \%$. [4, 5, 7, $8,11,12,16-19]$. In published studies the preparation time seems to have an important influence on the cleaning result. Especially when PEG is used, longer preparation periods of 2 to 4 days have a tendency to yield better cleaning results $[10,17]$, while the rate of good to excellent results in 1-day regimens does not exceed $88 \%$ [19]. When sodium picosulphate is used, up to $100 \%$ of good to excellent cleaning is reported [18], but it should be noted that according to our results, no difference in terms of effectiveness could be found in 2 singlecenter randomized controlled trials directly comparing PEG and sodium picosulphate $[20,21]$. Taken together, our results accord reasonably well with published data if the typical short duration of the preparation period ( 1 day or less) in our patients is taken into account. We hypothesize that an extension of the preparation period and a close monitoring of bowel cleansing would lead to improved results but that cannot be derived from our data.

Studies investigating short preparation protocols report abdominal pain in up to $53 \%$ of patients [5] and nausea and vomiting in up to $60 \%$ [3]. When sodium picosulphate is used, the rate of AEs in single studies seems to be lower $[18,22]$. In direct comparison, sodium picosulphate was better tolerated than PEG $[20,21]$ with a significantly lower need for nasogastric tube insertion corresponding to our results [20].

The majority of our patients received PEG in combination with electrolytes. It should be noted that several authors have recognized low palatability of PEG preparations with electrolytes as an issue. In 1 study nasogastric tubes were necessary in the majority of children [6]. Thus the majority of studies in recent years have used electrolyte-free PEG preparations [3,8,10-12,17,23].
Nevertheless, no clear difference in the occurrence of AEs between electrolyte-containing and electrolyte-free solutions can be deduced from data in the literature. Abdominal pain has been reported in $18 \%$ to $53 \%$ versus $23 \%$ to $44 \%$ of patients, nausea and vomiting in $24 \%$ to $40 \%$ versus $16 \%$ to $60 \%$ of electrolyte-containing versus electrolyte-free solutions, respectively [3-6,8]. Completion rates have only been reported in a few studies and also have shown comparable results $[3,7]$. There is a lack of direct comparisons between the 2 types of PEG preparations. In our study only 9 of the 317 patients who received PEG as part of their preparation regimen were prepared with electrolyte-free PEGsolution. This is probably due to the fact that no such cleansing agent with official approval for colon preparation is available in Germany. In 2 of the 9 patients the solution was applied via a nasogastric tube, a rate that corresponds well with the rest of our sample.

Oral sodium phosphate was administered to $9.2 \%$ of our patients. The use of oral sodium phosphate in children is limited due to the potential risk of electrolyte imbalance and fatal hyperphosphatemia and the fact that it can cause hemorrhagic gastropathy, erosions, and histologic mucosal changes [24-26]. In our data we detected a 2-fold risk of vomiting when sodium phosphate was used. No further differences in comparison with other agents could be ascertained.

Our analysis is the first one of its kind in Germany. Its strengths are the large number of colonoscopies analyzed and the structured data collection on the basis of single endoscopies. The mix of participants represents a balanced sample, including pediatric gastroenterologists at all healthcare levels. The cleaning effect as the most relevant dependent variable was recorded on the basis of a validated score and not only by subjective assessment, even though the use of the Aronchick score was neither practiced prior to implementation by participants nor independently controlled. The limitations of this study result primarily from its open observational design. This was not a controlled trial and there was no randomization or standardization of the conditions of the bowel preparation. Some parameters that were probably relevant could not be recorded with reasonable effort. For example, we were able to analyze the elements of the different regimens but neither precise dosages nor details of the application (e.g. controlling the cleaning effect during preparation by parents and nurses) could be recorded. We are not able to be more precise concerning the influence of the type of indication, because our participating centers could only choose from the fixed categories in our database. Furthermore, sedation practices were not part of our survey. They are known to vary highly in Germany due to differences in local resources and personal preferences of the performing endoscopists and due to the lack of evidence based guidelines. Differences within the groups of patients (selection bias) or differences in the examiner's personal way of applying the Aronchick score (interobserver bias) could be additional confounding factors. Thus we cannot exclude that relevant influencing factors were omitted from our analysis. Despite these limitations we were able to provide novel data regarding the effectiveness, tolerability, and safety of bowel preparation regimens in children.

In conclusion, in our sample of 768 colonoscopies, we found a large variety of regimens used for bowel preparation, the most frequently used cleansing agents being sodium picosulphate and PEG. Our analysis points to a strongly increased need for the use of nasogastric tubes, if PEG is used for bowel preparation in children and adolescents prior to colonoscopy, whereas the different 
agents seem to have no discernible influence on the cleaning result. Sodium picosulphate apparently has comparable effectiveness and proves to be better tolerated. Further well-designed direct comparisons between the 2 cleansing agents will be the logical next step to further promote the ongoing search for an ideal regimen for children.

\section{Competing interests: None}

Institutions

${ }^{1}$ Vestische Kinder- und Jugendklinik Datteln, University of Witten-Herdecke, Datteln, Germany

2 Department of Child and Adolescent Medicine, Klinikum Links der Weser, Bremen, Germany

${ }^{3}$ Kinderkrankenhaus Sankt Marien, Landshut, Germany

${ }^{4}$ Division of Child and Adolescent Medicine, Deutsche Klinik für Diagnostik, Wiesbaden, Germany

${ }^{5}$ Department of Child and Adolescent Medicine, University Hospital Carl Gustav Carus, Technische Universität Dresden, Dresden, Germany

${ }^{6}$ Praxis für Kinder und Jugendliche, Kassel, Germany

${ }^{7}$ Department of Pediatrics and Adolescent Medicine, University Medical Center Ulm, Ulm, Germany

${ }^{8}$ Christliches Kinderhospital, Osnabrück, Germany

${ }^{9}$ Institute of Medical Biometry and Epidemiology, University of WittenHerdecke, Witten, Germany

${ }^{10}$ Department of Newborn, Child and Adolescent Medicine, Klinikum Nürnberg, Nürnberg, Germany

\section{Acknowledgements}

$\nabla$

We appreciate the participation and cooperation of the following colleagues:

Rolf Behrens, Department of Newborn, Child and Adolescent Medicine, Klinikum Nürnberg

Thomas Berger, Vestische Kinder- und Jugendklinik, University of Witten-Herdecke, Datteln*

Stephan Buderus, Klinik für Kinder- und Jugendmedizin, GFO Kliniken Bonn

Martin Burdelski, Klinik für Allgemeine Pädiatrie, Universitätsklinikum Schleswig-Holstein, Campus Kiel*

Martin Classen, Department of Child and Adolescent Medicine, Klinikum Links der Weser, Bremen

Söhnke Dammann, Klinik für Kinder- und Jugendmedizin, Oberschwabenklinik, Ravensburg

Gesche Düker, Abteilung für Allgemeine Pädiatrie, Unversitätsklinikum, Bonn*

Harald Engelhardt, Kinderkrankenhaus Sankt Marien, Landshut Guido Engelmann, Zentrum für Kinder- und Jugendmedizin,

Universitätsklinikum, Heidelberg*

Thorsten Fröhlich, Kinderklinik, Universitätsklinikum Erlangen* Ulrich Gabel, Praxis für Kinder- und Jugendmedizin Oberursel Matthias Heiduk, Klinik für Kinder- und Jugendmedizin,

Klinikum Magdeburg

Christoph Herder, DRK-Kinderklinik Siegen

Rüdiger Kardorff, Zentrum für Kinder- und Jugendliche, Marienhospital Wesel

Klaus-Michael Keller, Division of Child and Adolescent Medicine, Deutsche Klinik für Diagnostik, Wiesbaden

Ute Kloß, Kinder- und Jugendmedizin, Vivantes Klinikum im

Friedrichshain Berlin

Andreas Krahl, Darmstädter Kinderkliniken Prinzessin Margaret,

Darmstadt

Benno Kretzschmar, Klinik für Kinder- und Jugendmedizin
“Dr. Siegfried Wolff”, Eisenach

Martin Laaß, Department of Child and Adolescent Medicine, University Hospital Carl Gustav Carus, Technische Universität Dresden*

Thomas Lang, Kinder- und Jugendmedizin, Krankenhaus Barmherzige Brüder, Regensburg*

Werner Luck, Charité-Campus Virchow-Kliniken Otto-Heubner-

Centrum für Kinder- und Jugendmedizin, Berlin*

Ralph Melchior, Praxis für Kinder und Jugendliche, Kassel Carsten Posovszky, Department of Pediatrics and Adolescent Medicine, University Medical Center Ulm*

Olaf Raecke, Klinik für Kinder und Jugendliche, Klinikum Esslingen

Burkhard Rodeck, Christliches Kinderhospital, Osnabrück Anjona Schmidt-Choudhury, Klinik für Kinder- und Jugendmedizin, Katholisches Klinikum Bochum*

Ralf Seul, Kinder- und Jugendmedizin, Marienhospital Witten Ulf Winkler, Klinik für Kinder- und Jugendmedizin, OberlausitzKliniken Bautzen

\section{References}

1 Rex DK, Imperiale TF, Latinovic DR et al. Impact of bowel preparation on efficiency and cost of colonoscopy. Am J Gastroenterol 2002; 97: $1696-1700$

2 Steffen RM, Wyllie R, Sivak MV et al. Colonoscopy in the pediatric patient. J Pediatr 1989; 115: $507-514$

3 Abbas MI, Nylund CM, Bruch CJ et al. Prospective evaluation of 1-day polyethylene glycol-3350 bowel preparation regimen in children. J Pediatr Gastroenterol Nutr 2013; 56: 220-224

4 Da Silva MM, Briars GL, Patrick MK et al. Colonoscopy preparation in children: safety, efficacy, and tolerance of high- versus low-volume cleansing methods. J Pediatr Gastroenterol Nutr 1997; 24: 33 - 37

5 Gremse DA, Sacks AI, Raines S. Comparison of oral sodium phosphate to polyethylene glycol-based solution for bowel preparation for colonoscopy in children. J Pediatr Gastroenterol Nutr 1996; 23: 586- 590

6 Sondheimer JM, Sokol RJ, Taylor SF et al. Safety, efficacy, and tolerance of intestinal lavage in pediatric patients undergoing diagnostic colonoscopy. J Pediatr 1991; 119: 148-152

7 Terry NA, Chen-Lim ML, Ely E et al. Polyethylene glycol powder solution versus senna for bowel preparation for colonoscopy in children. J Pediatr Gastroenterol Nutr 2013; 56: 215-219

8 Walia $R$, Steffen $R$, Feinberg $L$ et al. Tolerability, safety, and efficacy of PEG 3350 as a 1-day bowel preparation in children. J Pediatr Gastroenterol Nutr 2013; 56: 225 - 228

9 Hunter A, Mamula P. Bowel preparation for pediatric colonoscopy procedures. J Pediatr Gastroenterol Nutr 2010; 51: 254-261

10 Jibaly $R$, LaChance J, Lecea NA et al. The utility of PEG3350 without electrolytes for 2-day colonoscopy preparation in children. Eur J Pediatr Surg 2011; 21: 318-321

11 Kierkus J, Horvath A, Szychta M et al. High- versus low-volume polyethylene glycol plus laxative versus sennosides for colonoscopy preparation in children. J Pediatr Gastroenterol Nutr 2013; 57: $230-235$

12 Phatak UP, Johnson S, Husain SZ et al. Two-day bowel preparation with polyethylene glycol 3350 and bisacodyl: a new, safe, and effective regimen for colonoscopy in children. J Pediatr Gastroenterol Nutr 2011; 53: $71-74$

13 Turner D, Levine A, Weiss B et al. Evidence-based recommendations for bowel cleansing before colonoscopy in children: a report from a national working group. Endoscopy 2010; 42: 1063-1070

14 Aronchick C, Lipshutz W, Wright $S$ et al. Validation of an instrument to assess colon cleansing. Am J Gastroenterol 1999; 94: 2667

15 ASGE Standards of Practice Committee. Saltzman JR, Cash BD, Pasha SF et al. Bowel preparation before colonoscopy. Gastrointest Endosc 2015; 81: 781 - 794

16 Kawakami E, Portorreal A, Scuissiatto ML et al. [Bowel preparation for colonoscopy with sodium picosulphate and magnesium citrate in children and adolescents]. Arq Gastroenterol 2004; 41: 33 - 36

17 Pashankar DS, Uc A, Bishop WP. Polyethylene glycol 3350 without electrolytes: a new safe, effective, and palatable bowel preparation for colonoscopy in children. J Pediatr 2004; 144: 358-362

\footnotetext{
* academic centers
} 
18 Pinfield A, Stringer MD. Randomised trial of two pharmacological methods of bowel preparation for day case colonoscopy. Arch Dis Child 1999; 80: 181 - 183

19 Shaoul R, Haloon L. An assessment of bisacodyl-based bowel preparation for colonoscopy in children. J Gastroenterol 2007; 42: 26 - 28

20 Turner D, Benchimol EI, Dunn H et al. Pico-Salax versus polyethylene glycol for bowel cleanout before colonoscopy in children: a randomized controlled trial. Endoscopy 2009; 41: 1038 - 1045

21 Di Nardo G, Aloi M, Cucchiara S et al. Bowel preparations for colonoscopy: An RCT. Pediatrics 2014; 134: 249-256

22 Jimenez-Rivera C, Haas D, Boland $M$ et al. Comparison of two common outpatient preparations for colonoscopy in children and youth. Gastroenterol Res Pract 2009; 2009: 518932
23 Safder S, Demintieva $Y$, Rewalt $M$ et al. Stool consistency and stool frequency are excellent clinical markers for adequate colon preparation after polyethylene glycol 3350 cleansing protocol: a prospective clinical study in children. Gastrointest Endosc 2008; 68: 1131 - 1135

24 Hassall E, Lobe TE. Risky business: oral sodium phosphate for precolonoscopy bowel preparation in children. J Pediatr Gastroenterol Nutr 2007; 45: $268-269$

25 Lawrance IC, Willert RP, Murray K. Bowel cleansing for colonoscopy: prospective randomized assessment of efficacy and of induced mucosal abnormality with three preparation agents. Endoscopy 2011; 43: $412-418$

26 Nam SY, Choi IJ, Park KW et al. Risk of hemorrhagic gastropathy associated with colonoscopy bowel preparation using oral sodium phosphate solution. Endoscopy 2010; 42: 109-113 